\title{
Selection of microRNAs in extracellular vesicles for diagnosis of malignant pleural mesothelioma by in vitro analysis
}

\author{
TAKANOBU JOTATSU ${ }^{1}$, HIROTO IZUMI ${ }^{2}$, YASUO MORIMOTO ${ }^{2}$ and KAZUHIRO YATERA ${ }^{1}$ \\ ${ }^{1}$ Department of Respiratory Medicine, and ${ }^{2}$ Department of Occupational Pneumology, Institute of \\ Industrial Ecological Sciences, University of Occupational and Environmental Health, Kitakyushu, Fukuoka 807-8555, Japan
}

Received April 9, 2020; Accepted September 4, 2020

DOI: $10.3892 /$ or.2020.7778

\begin{abstract}
Malignant pleural mesothelioma (MPM) is a malignant tumor which is a challenge for diagnosis and is associated with a poor patient prognosis. Thus, early diagnostic interventions will improve the quality of life and life expectancy of these patients. Recently, cellular microRNAs (miRNAs) have been found to be involved in maintaining homeostasis, and abnormal miRNA expression has often been observed in various diseases including cancer. Extracellular vesicles (EVs) released by many cells contain proteins and nucleic acids. miRNAs are secreted from all cells via EVs and circulate throughout the body. In this study, culture media were passed sequentially through membrane filters 220-50 nm in size, and EVs with diameters of 50 to $220 \mathrm{~nm}$ (EVcap50/220) were collected. miRNAs (EV50-miRNAs) in EVcap50/220 were purified, and microarray analysis was performed. EV50-miRNA expression profiles were compared between MPM cells and a normal pleural mesothelial cell line, and six EV50-miRNAs were selected for further investigation. Of these, hsa-miR-193a-5p and hsa-miR-551b-5p demonstrated higher expression in MPM-derived EVcap50/220. These miRNAs reduced the expression of several genes involved in cell-cell interactions and cell-matrix interactions in normal pleural mesothelial cells. Our data suggest that hsa-miR-193a-5p and hsa-miR-551b-5p in EVcap50/220 could be diagnostic markers for MPM.
\end{abstract}

\section{Introduction}

Malignant pleural mesothelioma (MPM) is a rare malignancy that originates from the pleural mesothelium and is associated with an extremely poor patient prognosis. MPM is strongly

Correspondence to: Dr Hiroto Izumi, Department of Occupational Pneumology, Institute of Industrial Ecological Sciences, University of Occupational and Environmental Health, 1-1, Iseigaoka, Yahata-nishi-ku, Kitakyushu, Fukuoka 807-8555, Japan

E-mail: h-izumi@med.uoeh-u.ac.jp

Key words: malignant pleural mesothelioma, extracellular vesicle, microRNA, miR-193a-5p, miR-551b-5p associated with asbestos exposure (1) and has a long latency period (20-40 years) following asbestos exposure (2). Asbestos has been banned in Japan since 2006, but there are countries where asbestos is still mined and used. The incidence of MPM has increased over the last decade and is predicted to peak sometime before 2030 (2). Moreover, the death toll from asbestos exposure is expected to rise. The symptoms of MPM include chest tightness, shortness of breath, and coughing; these symptoms are often caused by pleural effusion. Unfortunately, MPM frequently does not show early symptoms, and therefore, the disease is often progressed by the time it is diagnosed. Therefore, it is extremely important to diagnose MPM in its early stages.

Diagnosing MPM is often difficult. Comprehensive diagnosis consists of biopsy of the suspected tumor lesion and measuring pleural effusion. In the case of epithelioid MPM, a cytological diagnosis can be conducted by examining pleural effusion (3). In contrast, sarcomatoid MPM is very difficult to diagnose via pleural effusion examination. Secondary markers of MPM, such as mesothelin (4), osteopontin (5), and fibulin-3 (6) have been reported. However, they are not necessarily reliable diagnostic markers due to their variability in values. Therefore, there are still no useful markers for MPM diagnosis.

MicroRNAs (miRNAs/miRs) are small non-coding RNAs (approximately 20-22 nucleotides) that function in diverse biological processes and regulate the expression of target genes. Many researchers have reported that miRNAs are associated with cancer development, progression, and metastasis (7-9). In MPM, miRNAs have recently gained attention as diagnostic and therapeutic targets. For example, it has been reported that the miR-200 family can be as potential candidates with which to differentiate MPM from other cancers in a diagnostic setting (10).

Extracellular vesicles (EVs) include exosomes, microvesicles, and apoptotic bodies. EVs consist of a lipid bilayer that envelops diverse proteins, mRNAs, and miRNAs. In terms of diameter, exosomes range between 20 and $150 \mathrm{~nm}$, microvesicles between $100 \mathrm{~nm}$ and $1 \mu \mathrm{m}$, and apoptotic bodies are more than $1 \mu \mathrm{m}$. While exosomes and microvesicles are produced differently, both are secreted from intact cells and circulate throughout the body (11). Therefore, EVs can be found in any fluid (blood, saliva, urine, ascites fluid and pleural effusion). Exosomes and microvesicles are taken up by other cells via 
endocytosis, and it has been shown that miRNAs contained in EVs function in cells that they are taken up by (12-15). These findings have garnered a wave of attention towards functionalized EVs as intercellular transmitters.

There is not yet a standard purification method to individually isolate exosomes and microvesicles. For EV purification, ultracentrifugation, exosome capturing using proteins expressed on the exosome membrane, and size exclusion chromatography are used. However, separating exosomes and microvesicles may be difficult. In this study, we purified EVs containing exosomes and microvesicles in a diameter-dependent manner with membrane filters. This method is simple and easy. Using this method, six miRNAs in EVs that were secreted by MPM cells but not normal mesothelial cells were selected. We believe that these miRNAs are promising targets for MPM diagnosis via blood and pleural effusion measurements.

\section{Materials and methods}

Cell culture. We used six malignant pleural mesothelioma (MPM) cell lines (ACC-MESO1, ACC-MESO4, MSTO-211H, L324, N407, and K921) and a normal pleural mesothelial cell line (MeT-5A). ACC-MESO1 and ACC-MESO4 were obtained from the Riken Cell Bank (Tsukuba, Japan). MSTO-211H was purchased from the American Type Culture Collection (ATCC, Manassas, VA, USA). L324, N407, and K921 cells were generated in the laboratory and kindly donated by Dr Hidetaka Uramoto, Kanazawa Medical University, Ishikawa (16). MeT-5A cells were purchased from ATCC. Human prostate cancer PC3 cells were obtained as previously described (17). Immortalized human pulmonary fibroblast (IHPF) cells (T0490) were purchased from Applied Biological Materials Inc. (Richmond, BC, Canada). MPM, MeT-5A, PC3, and IHPF cells were maintained in RPMI-1640 medium, Medium 199, DMEM (Thermo Fisher Scientific, Inc.), and Prigrow III medium (Applied Biological Materials Inc.), respectively. All cells were cultured with GlutaMAX supplement (Thermo Fisher Scientific, Inc.), 10\% heat-inactivated fetal bovine serum (FBS), and $1 \%$ (v/w) penicillin/streptomycin, and were maintained at $37^{\circ} \mathrm{C}$ in $5 \% \mathrm{CO}_{2}$.

Preparation of GFP/Nluc-fused CD9, CD63, CD81, hsa-miR-4728-5p, hsa-miR-193a-5p, and hsa-miR-551b-5p expression plasmids. CD63-green fluorescent protein (GFP) (CYTO122-PA-1), CD9-GFP (CYTO120-PA-1), and CD81-red fluorescent protein (RFP) (CYTO125-PA-1) expression plasmids were purchased from System Biosciences. The pNL1.1 vector containing nanoluciferase (Nluc) was purchased from Promega Corp. CD63-Nluc, CD9-Nluc, CD81-Nluc, and CD81-GFP expression plasmids were constructed with these plasmids. GFP fusion proteins were used for qualitative analysis of intracellular expression and intracellular uptake. Nluc fusion proteins were used for quantitative analysis of extracellular secretion and intracellular uptake. Double-stranded oligonucleotides against hsa-miR-4728-5p, hsa-miR-193a-5p, and hsa-miR-551b-5p were synthesized (Table SI) and ligated into multicloning sites of pSIH1-H1-GFP-T2A-Puro, which was originally constructed with pSIH1-H1-copGFP (SI501A-1, System Biosciences) and pCDH-EF1-MCS-BGH-PGK-GFP-T 2A-Puro (CD550A-1, System Biosciences).
Expression by the lentivirus system. Lentivirus-containing expression cassettes of GFP/Nluc fusion genes and miRNAs were obtained using the System Biosciences lentivirus packaging system. Lentiviral transduction was performed according to the manufacturer's protocol. Briefly, after infecting the MeT-5A cells, cells expressing CD63-GFP/Nluc, CD9-GFP/Nluc, CD81-GFP/Nluc were selected using $10 \mu \mathrm{g} / \mathrm{ml}$ of puromycin. In the same way, MeT-4728-5p, MeT-193a-5p and MeT-551b-5p cells expressing hsa-miR-4728-5p, hsa-miR-193a-5p and hsa-miR-551b-5p, respectively, were obtained by infection and selection with MeT-5A cells. MeT-ctrl cells did not express miRNA. Fused GFPs or independently expressed GFP of the Nluc fusion protein and miRNAs were observed using an EVOS fluorescence microscope (Thermo Fisher Scientific, Inc.). After confirming that over $90 \%$ of the cells were expressing GFP using LUNA-FL ${ }^{\mathrm{TM}}$ (Logos Biosystems, Korea), they were used in the assay.

Isolation and removal of EVs. Syringe filters with sizes of $220 \mathrm{~nm}$ (SFPES013022N), $50 \mathrm{~nm}$ (SF16008), and $20 \mathrm{~nm}$ (2.CF7103.0001) were purchased from Membrane Solutions, TISCH Scientific, and ANPEL Laboratory Technologies, respectively. In our laboratory, FBS was passed through a 220-nm bottle-top vacuum filter (Thermo Fisher Scientific, Inc.) (FBSpas220) to maintain normal cells. To remove EVs from FBS, FBSpas220 was passed through 50-nm syringe filters (FBSpas50). When cells grown in 10-cm dishes containing maintenance medium were $80 \%$ confluent, they were collected and seeded into three $10-\mathrm{cm}$ dishes containing FBSpas50. After $48 \mathrm{~h}$, the culture medium was collected and passed sequentially through $220 \mathrm{~nm}$ and $50 \mathrm{~nm}$ syringe filters. EVs captured by the $50 \mathrm{~nm}$ syringe filter (EVcap50/220) were partially collected by reverse-flow with phosphate-buffered saline (PBS) (EVrev50/220). The EVrev50/220 and EVs that had passed through a $50 \mathrm{~nm}$ syringe filter (EVpas50) were analyzed by dynamic light scattering (DLS, Zetasizer, Malvern Panalytical, UK) and transmission electron microscopy (TEM, JEOL 1200EX; JEOL, Japan). EVcap50/220 was used directly for RNA extraction and protein extraction, as outlined in the procedures below.

Dynamic light scattering (DLS) and transmission electron microscopy (TEM) were performed. The diameter of EVs obtained by reverse flow was measured by DLS (Zetasizer) according to the manufacturer's instructions, and as previously described (18). Samples were prepared using an Exosome TEM-easy kit (101 Bio, Mountain View, CA, USA) with EVs obtained from reverse flow; observation was carried out on a TEM (JEOL 1200EX).

Cell proliferation assay. PC3 cells (1,000 cells) were seeded into 12-well plates. Cell number was measured the next day and was used as the baseline at $0 \mathrm{~h}$. PC3 cells were washed twice with PBS, and were added to either medium without FBS, medium containing FBSpas220 (normal medium), or medium containing FBSpas50. Cells were collected every $24 \mathrm{~h}$, and the number of cells was calculated by LUNA-FL ${ }^{\mathrm{TM}}$ (Logos Biosystems) according to manufacturer's instructions. Experiments were performed in triplicates. 
Protein extraction and western blot analysis. When MeT-5A cells grown in $10-\mathrm{cm}$ dishes containing maintenance medium were $50 \%$ confluent, they were washed twice with PBS, and added with FBSpas50-containing medium. After $48 \mathrm{~h}$, cultured medium or fresh medium containing FBSpas50 (for the negative control) was collected and passed sequentially through 220 and $50 \mathrm{~nm}$ syringe filters. Proteins of EVcap50/220 were eluted with $2 \mathrm{ml}$ CHAPS lysis buffer (Dojindo) containing $1 \mathrm{mM}$ PMSF. Each eluted solution was concentrated using Amicon Ultra $10 \mathrm{kDa}$ (Merck Millipore) until its volume was less than $30 \mu 1$. All samples were subjected to sodium dodecyl sulfate-polyacrylamide gel electrophoresis (SDS-PAGE) and were transferred to polyvinylidene difluoride (PVDF) membranes. The membrane was immunoblotted first with anti-EpCAM antibody (EBA-1, dilution 1:1,000, Santa Cruz Biotechnology, Inc.), CD63 antibody (TS63, dilution 1:1,000, Thermo Fisher Scientific, Inc.), CD9 antibody (TS9, dilution 1:1,000, Thermo Fisher Scientific, Inc.), CD81 antibody (M38, dilution 1:1,000, Thermo Fisher Scientific, Inc.). Secondary antibodies conjugated with horseradish peroxidase were used for detection. Signal intensity was obtained using LAS 4000 Mini and Multi Gauge software version 3.0 (Fujifilm), as previously described (17).

Expression of GFP/Nluc fusion protein and analysis of extracellular secretion. MeT-5A cells expressing and secreting CD9-GFP/Nluc, CD63-GFP/Nluc, and CD81-GFP/Nluc were cultured in plates or dishes with medium containing FBSpas50. These cells are called donor cells because the fusion proteins secreted by these cells are taken up by other cells. After the indicated hours, cultured medium or cells were collected. Each medium was passed through a $220 \mathrm{~nm}$ or $50 \mathrm{~nm}$ syringe filter and was used for Nluc assays. Cells were washed twice with PBS and lysed with Reporter Lysis Buffer (Promega Corp.). The cell lysate was vortexed, centrifuged (21,000 x g for $2 \mathrm{~min})$, and the supernatant was collected for Nluc assays. Nluc activity was measured using the Nano-Glo ${ }^{\circledR}$ Luciferase Assay System (Promega Corp.) according to the manufacturer's instructions. In order to observe secreted EVs with the GFP fusion protein via an EVOS fluorescence microscope (Thermo Fisher Scientific, Inc.), EVs were concentrated using a Total Exosome Isolation kit (Thermo Fisher Scientific, Inc.) according to the manufacturer's instructions.

Efficiency of EV capture by membrane filters. Culture medium of MeT-5A cells that expressed CD81-Nluc was passed through a 220-nm syringe filter (EVpas220). EVpas50/220 and EVpas20/50 were obtained by passing EVpas220 through a $50-\mathrm{nm}$ syringe filter and by passing the EVpas50/220 through a 20-nm syringe filter, respectively. Nluc activities of EVpas220, EVpas50/220, and EVpas20/50 were measured as described above.

Uptake assay of the GFP/Nluc fusion protein. To obtain the GFP fusion protein, MeT-5A cells expressing CD9-GFP, CD63-GFP, and CD81-GFP (donor cells) were cultured with $10 \mathrm{ml}$ Opti-MEM medium without FBS for $48 \mathrm{~h}$. Each culture medium was passed through a $220-\mathrm{nm}$ syringe filter and concentrated to $30 \mu \mathrm{l}$ or less by Amicon Ultra-4 (Merck Millipore). They were added to the culture medium of recipient cells that were able to take up the GFP fusion protein. After $8 \mathrm{~h}$, cells were washed twice with PBS, and were observed under an EVOS fluorescence microscope (Thermo Fisher Scientific, Inc.). For the Nluc fusion protein, MeT-5A cells expressing CD81-Nluc were cultured in medium containing FBSpas50. After $48 \mathrm{~h}$, each culture medium was passed through 220 and 50-nm syringe filters, and was independently replaced with media of recipient cells. After the allocated hours, Nluc activities in cell lysates were measured as described above, and were standardized to cell protein concentration. Uptake of EVs with diameters between 50 and $220 \mathrm{~nm}$ was determined by subtraction.

RNA extraction. The medium that passed through the $220-\mathrm{nm}$ syringe filter was passed through a $50-n m$ syringe filter to obtain EVcap50/220. Total RNA of EVcap50/220 was eluted with $1 \mathrm{ml}$ ISOGEN (Nippongene). The eluted solution was mixed with $200 \mu \mathrm{l}$ chloroform, and was centrifuged at $12,000 \mathrm{x} \mathrm{g}$ for $15 \mathrm{~min}$. Total RNA was purified with $500 \mu 1$ supernatant using a NucleoSpin ${ }^{\circledR}$ miRNA Plasma Kit (Macherey-Nagel), which was used according to the manufacturer's instructions. Total cell RNA was eluted in $100 \mu 1 \mathrm{H}_{2} \mathrm{O}$, and was precipitated with $10 \mu \mathrm{l}$ of $3.0 \mathrm{M}$ potassium acetate, $100 \mu \mathrm{l}$ isopropanol, and $1 \mu \mathrm{l}$ Pellet Paint. After washing and drying the pellet, RNA was dissolved in $2 \mu \mathrm{l}$ or $10 \mu 1 \mathrm{H}_{2} \mathrm{O}$ for microarray or quantitative real-time polymerase chain reaction (qPCR), respectively. Verification of microRNA purification was performed with Eukaryote Total RNA Nano Series II using an Agilent 2100 Bioanalyzer (Agilent Technologies, Inc.). All RNAs, including the miRNAs from cells, were purified using the miRNeasy Mini Kit(Qiagen), according to the manufacturer's instructions.

Microarray. Microarray analyses with the Human miRNA Oligo chip-4 plex (TORAY, Tokyo, Japan) containing 2,565 probe sets and the Human Oligo chip 25k (TORAY) containing 24,460 probe sets were carried out using a 3D-Gene array system, according to the manufacturer's protocol. For the miRNA array, $2 \mu 1$ RNA solution obtained from $30 \mathrm{ml}$ medium or 250 ng total cell RNA were hybridized with the Human miRNA Oligo chip. For the mRNA array, $1 \mu \mathrm{g}$ total cell RNA was used to hybridize the Human Oligo Chip 25k.

$Q$ uantitative real-time polymerase chain reaction ( $q P C R)$. For RNA obtained from EVcap50/220, the dry pellet was dissolved with $10 \mu \mathrm{l}$ of $\mathrm{H}_{2} \mathrm{O}$, and $1 \mu \mathrm{l}$ RNA was used for each probe. For RNA obtained from cell lysates, RNA was prepared according to the manufacturer's instructions. cDNA for the miRNA was synthesized using the TaqMan MicroRNA Reverse Transcription Kit (Thermo Fisher Scientific, Inc.) in a StepOne Plus real-time PCR system (Thermo Fisher Scientific, Inc.). qPCR was performed using a specifically designed TaqMan probe. Primer sequences used to analyze human miRNAs and gene expression via qPCR were provided by the following sources: miR-4728-5p, Thermo Fisher Scientific, Inc., 461811_ mat; miR-193a-5p, Thermo Fisher Scientific, Inc., 002281; miR-551b-5p, Thermo Fisher Scientific, Inc., 002346; U6, Thermo Fisher Scientific, Inc., 001973. The cycling conditions were as follows: Denaturing, hold at $95^{\circ} \mathrm{C}$ for $20 \mathrm{sec}, 40$ cycles of amplification (denaturation at $95^{\circ} \mathrm{C}$ for $30 \mathrm{sec}$, annealing and extension at $60^{\circ} \mathrm{C}$ for $30 \mathrm{sec}$ ). U6 was used as an internal 
A

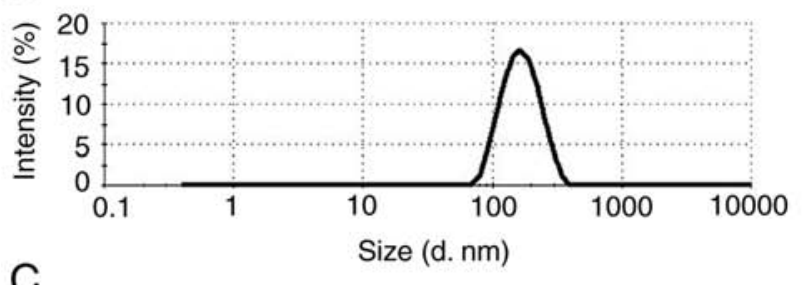

C

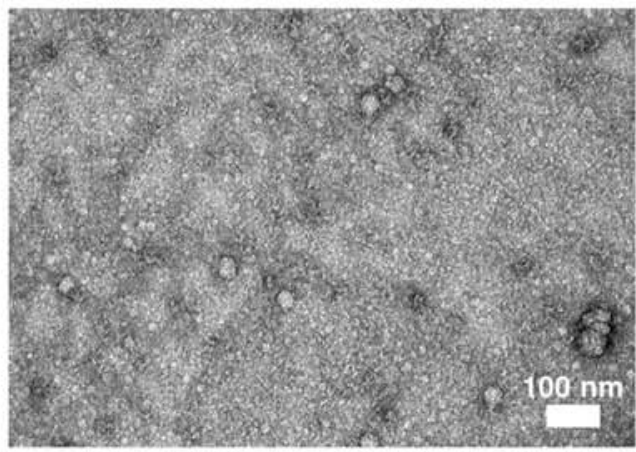

E

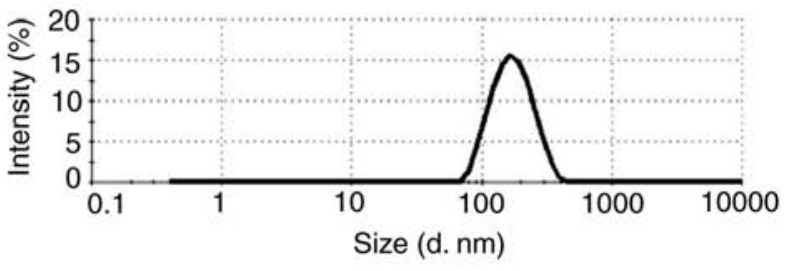

B

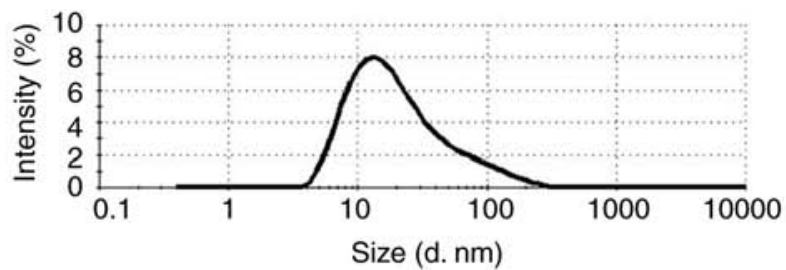

D
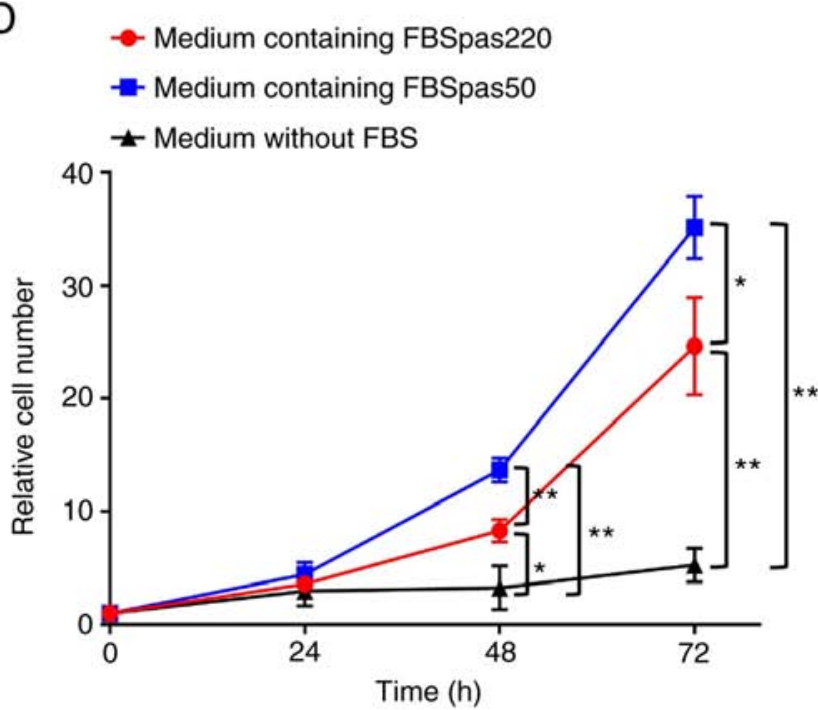

F

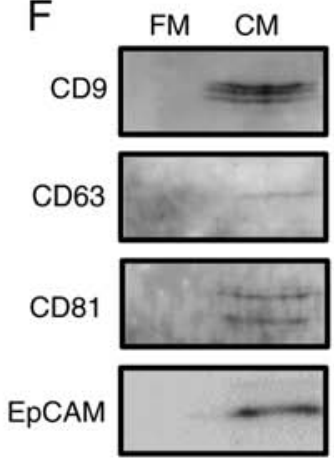

G

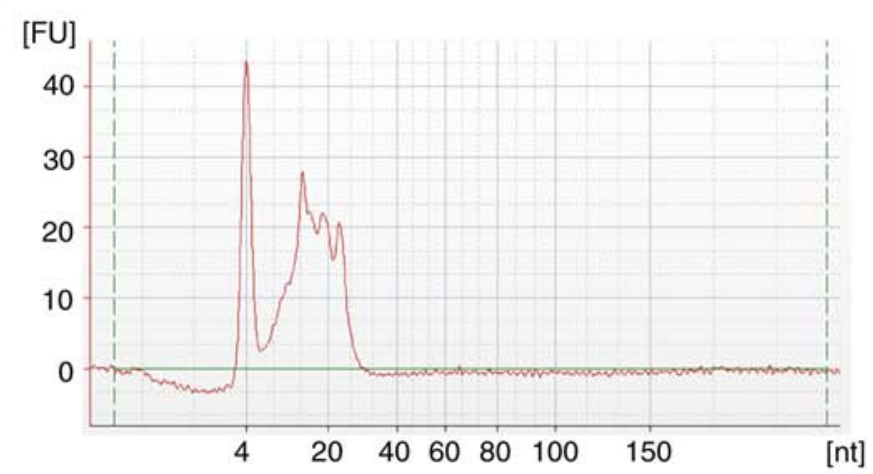

Figure 1. Characterization of EVs derived from fetal bovine serum (FBS). (A) EVs captured with a 50-nm filter (EVcap50/220) were collected by reverse flow (EVrev50/220), and their size was analyzed by dynamic light scattering (DLS). (B) A flow-through 50-nm filter (EVpas50/220) was also analyzed by DLS. (C) EVrev50/220 were observed using a transmission electron microscope (TEM). (D) PC3 cells were cultured in indicated media and cell numbers were counted after the indicated number of hours. The results were normalized to cell numbers at $0 \mathrm{~h}$. Points, mean of at least three independent experiments; bars, SD. ${ }^{*} \mathrm{P}<0.05{ }^{* * *} \mathrm{P}<0.01$. The experiment was performed three times independently, and cell number at $0 \mathrm{~h}$ was set to 1 . (E) EVrev50/220 of cell culture medium were collected and their size was analyzed by DLS. (F) Eluted proteins obtained from EVcap50/220 of each fresh medium (FM) and medium cultured for $48 \mathrm{~h}$ (CM) were subjected to SDS-PAGE, and western blotting was performed with anti-EpCAM, anti-CD9, anti-CD63, and anti-CD81 antibodies. (G) Total RNA purified from EVcap50/220 was analyzed by Bioanalyzer 2100. FU and nt indicate fluorescence units and nucleotides, respectively. EVs, extracellular vesicles.

control. The $\mathrm{Ct}$ values for each miRNA were normalized to $\mathrm{U6}$, and relative gene expression was calculated using the $\Delta \Delta \mathrm{Ct}$ method. All samples were run in duplicates in each experiment.

Statistics. Statistical analysis was performed using GraphPad Prism7 (GraphPad Software, Inc.). Results were compared using a two-tailed Student's t-test and one-way analysis of variance (ANOVA) followed by Turkey's test and Dunnett's test. Data are expressed as mean \pm SD. $\mathrm{P}<0.05$ was considered to indicate a statistically significant difference.

\section{Results}

Structural analysis of extracellular vesicles (EVs). In general, the cell culture medium was made by adding fetal bovine serum (FBS) to the basic culture medium. Since FBS contains EVs derived from bovine, it is necessary to remove them in order to analyze cultured cell-derived EVs. We usually use FBS passed through $220 \mathrm{~nm}$ (FBSpas220) for maintenance culture medium. In this experiment, to remove EVs that were under $50 \mathrm{~nm}$ in diameter, FBSpas220 were further passed through 


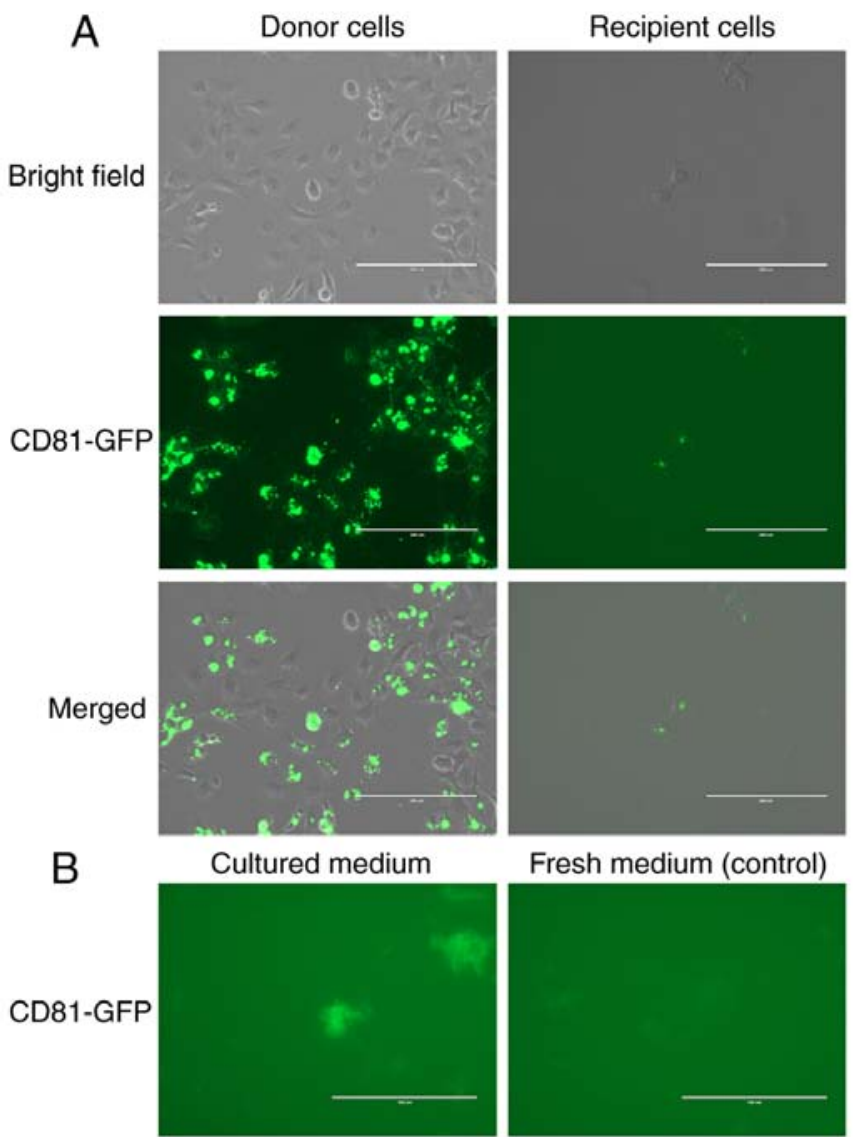

Figure 2. Expression, secretion, and uptake of CD81-GFP. (A) MeT-5A cells expressing CD81-GFP (donor cells) and MeT-5A cells uptaking EV expressing CD81-GFP (recipient cells) were observed under a fluorescence microscope. Bright field and GFP images were superimposed and shown as 'Merge'; length of the scale bar is $200 \mu \mathrm{m}$. (B) EVs concentrated from a medium containing EV expressing CD81-GFP (Cultured medium) or a medium not containing EV (Fresh medium) was observed with a fluorescence microscope. The length of the scale bar is $100 \mu \mathrm{m}$. EV, extracellular vesicle.

50-nm filters (FBSpas50). The efficiency of EV removal was analyzed via dynamic light scattering (DLS) with EVs captured by $50-\mathrm{nm}$ filters (EVcap50/220), as well as EVs that passed through those filters (EVpas50). As shown in Fig. 1A, the diameter of EVcap50/220 was approximately $170 \mathrm{~nm}$; however, this peak disappeared as they flowed through the 50-nm filters (Fig. 1B). To evaluate the shape of EVcap50/220, the EVs were collected by backflow (EVrev50/220). As shown in Fig. 1C, EVrev50/220 was spherical when analyzed using transmission electron microscopy (TEM). These results suggest that EVs from 50 to $220 \mathrm{~nm}$ diameter can be captured with $50 \mathrm{~nm}$ filters by passing the liquid sequentially through 220 and $50 \mathrm{~nm}$ filters (Fig. S1). Next, we investigated the effects of using FBSpas50 medium on cell growth. For this analysis, we used hormone-insensitive PC3 cells (19) with markedly rapid cell growth. As shown in Fig. 1D, cell proliferation was increased with a culture medium containing FBSpas50 when compared to FBSpas220-containing medium. This result suggested that FBSpas50 can be used for analysis of cultured cells, and that bovine-derived EVs inhibited cell growth. In this study, when isolating cell-derived EVs, cells were cultured in medium containing FBSpas50, and EVs secreted from the cells were captured with filters of various diameters. By this method,
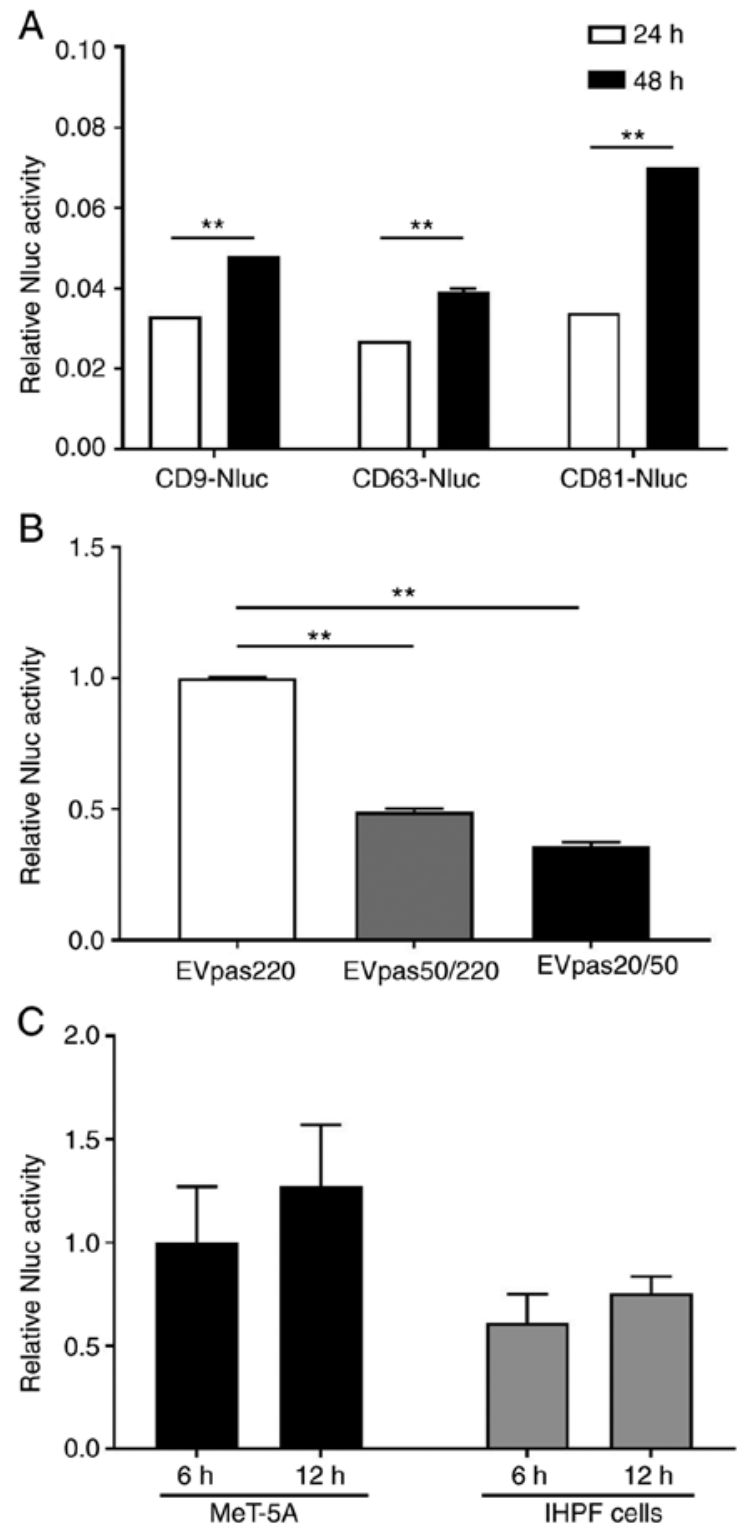

Figure 3. Expression, secretion, and uptake of CD81-Nluc. (A) MeT-5A cells expressing CD9-Nluc, CD63-Nluc, and CD81-Nluc were cultured with medium containing FBSpas50 for 24 and $48 \mathrm{~h}$. Nluc activity in each EVpas220 medium and cell lysate was measured. Nluc activity of each cell lysate was set to 1 . The experiment was performed three times independently; bars, SD. ${ }^{* *} \mathrm{P}<0.01$ vs. each 24 h. (B) MeT-5A cells expressing CD81-Nluc were cultured in medium containing FBSpas50 for $48 \mathrm{~h}$. Nluc activities of EVpas220, EVpas50/220 and EVpas20/50 were measured. Nluc activity of EVpas220 was set to 1. The experiment was performed three times independently; bars, SD. ${ }^{* *} \mathrm{P}<0.01$. (C) MeT-5A cells expressing CD81-Nluc were cultured in medium containing FBSpas50 for $48 \mathrm{~h}$. MeT-5A and IHPF cells were cultured with EVpas220 and EVpas50/220 for the indicated hours and each Nluc of cell lysate was measured. The value of Nluc activity with EVpas50/220 was subtracted from its value with EVpas220. Nluc activity of MeT-5A cells cultured for $6 \mathrm{~h}$ was set to 1 .

FBS-derived EVs are not contaminated during analysis with cell culture medium when filters greater than $50 \mathrm{~nm}$ are used.

MeT-5A cells were cultured in medium with FBSpas50 for $48 \mathrm{~h}$ and EVs in the medium were captured by $50-\mathrm{nm}$ filters. The diameter of EVcap50/220 was approximately $170 \mathrm{~nm}$ when measured by DLS (Fig. 1E). Proteins and RNAs of EVcap50/220 were analyzed. As shown in Fig. 1F, EVcap50/220 contained CD9, CD63, CD81, and EpCAM proteins, which are 
A
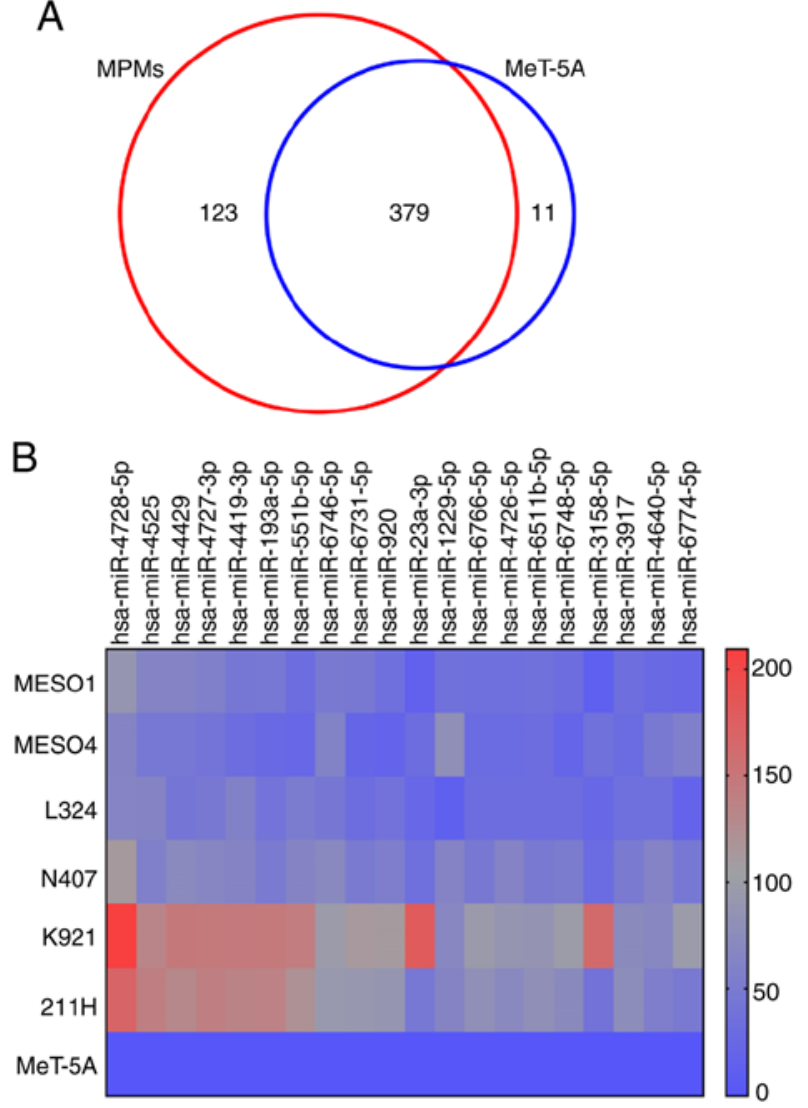

Figure 4. Comparison of EV50-miRNAs obtained from MeT-5A cells and six MPM cell lines. (A) Number of microRNAs commonly or specifically expressed, as shown by the Venn diagram. (B) Top 20 expression levels of EV50-miRNAs, as shown in a heat map. MPM, malignant pleural mesothelioma; EV50-microRNAs, microRNAs in extracellular vesicles captured by the 50 -nm filter.

markers of enriched exosomes. EVcap50/220 also contained microRNAs (miRNAs) less than $30 \mathrm{bp}$ in length (Fig. 1G). Particle diameter, shape, specific protein expression, and miRNA inclusion obtained from FBS and cell culture medium suggested that EVcap50/220 consisted of exosomes and microvesicles.

Functional analysis of the EVs. As EVs function as intercellular mediators, it is very important that EVs derived from cells (donor cells) are taken up by other cells (recipient cells). We generated MeT-5A cells that expressed CD9-green fluorescent protein (GFP), CD63-GFP, and CD81-GFP proteins, which are representative marker proteins of exosomes. Exosomes are membrane vesicles released by the fusion of organelles in the endocytic pathway, forming multivesicular bodies (20). Localization similar to multivesicular bodies was observed in cells expressing CD81-GFP (Fig. 2A, donor cells), CD9-GFP, and CD63-GFP (Figs. S2A and S3A). Next, we examined secretion of EVs containing CD81-GFP, CD9-GFP, and CD63-GFP. EVpas220 of culture medium was captured using the Total Exosome Isolation Kit as the EVpas220 was too small to be directly observed via a microscope. As shown in Fig. 2B, the GFP signal was observed in medium in which the cells expressed CD81-GFP, but not in the control medium. Results with CD9-GFP and CD63-GFP are shown
Table I. EV50-microRNAs expressed in 6 MPM cell lines but not expressed in EV50-microRNAs of MeT-5A cells (the 20 most common variants).

\begin{tabular}{lc}
\hline MicroRNA & Average expression in six MPM cell lines \\
\hline hsa-miR-4728-5p & 117.93 \\
hsa-miR-4525 & 83.58 \\
hsa-miR-4429 & 83.18 \\
hsa-miR-4727-3p & 82.95 \\
hsa-miR-4419b & 79.99 \\
hsa-miR-193a-5p & 73.78 \\
hsa-miR-551b-5p & 71.64 \\
hsa-miR-6746-5p & 69.19 \\
hsa-miR-6731-5p & 58.20 \\
hsa-miR-920 & 56.70 \\
hsa-miR-23a-3p & 54.11 \\
hsa-miR-1229-5p & 54.08 \\
hsa-miR-6766-5p & 52.48 \\
hsa-miR-4726-5p & 52.37 \\
hsa-miR-6511b-5p & 51.69 \\
hsa-miR-6748-5p & 50.54 \\
hsa-miR-3158-5p & 49.87 \\
hsa-miR-3917 & 49.03 \\
hsa-miR-4640-5p & 48.77 \\
hsa-miR-6774-5p & 48.22 \\
\hline
\end{tabular}

hsa, human (Homo sapiens); EV50-microRNAs, microRNAs in extracellular vesicles captured by the $50-\mathrm{nm}$ filter; MPM, malignant pleural mesothelioma.

Table II. EV50-microRNAs expressed in 6 MPM cell lines but not expressed in both EV50-microRNAs and cellular microRNAs of MeT-5A cells.

\begin{tabular}{lc}
\hline MicroRNA & Average expression in six MPM cell lines \\
\hline hsa-miR-193a-5p & 73.78 \\
hsa-miR-551b-5p & 71.64 \\
hsa-miR-3122 & 39.34 \\
hsa-miR-4771 & 24.62 \\
hsa-miR-195-3p & 17.62 \\
hsa-miR-3180-5p & 15.75
\end{tabular}

hsa, human (Homo sapiens); EV50-microRNAs, microRNAs in extracellular vesicles captured by the 50-nm filter; MPM, malignant pleural mesothelioma.

in Figs. S2B and S3B. These results suggested that the EVs contained CD63-GFP, CD9-GFP, and CD81-GFP. Since we observed that EVs contained GFP fusion protein in the culture medium, we investigated whether MeT-5A cells could take up EVs in the medium. As shown in Fig. 2A (recipient cells) and Figs. S2A and S3A, multivesicular bodies were observed $8 \mathrm{~h}$ after addition of the medium. These results suggested 

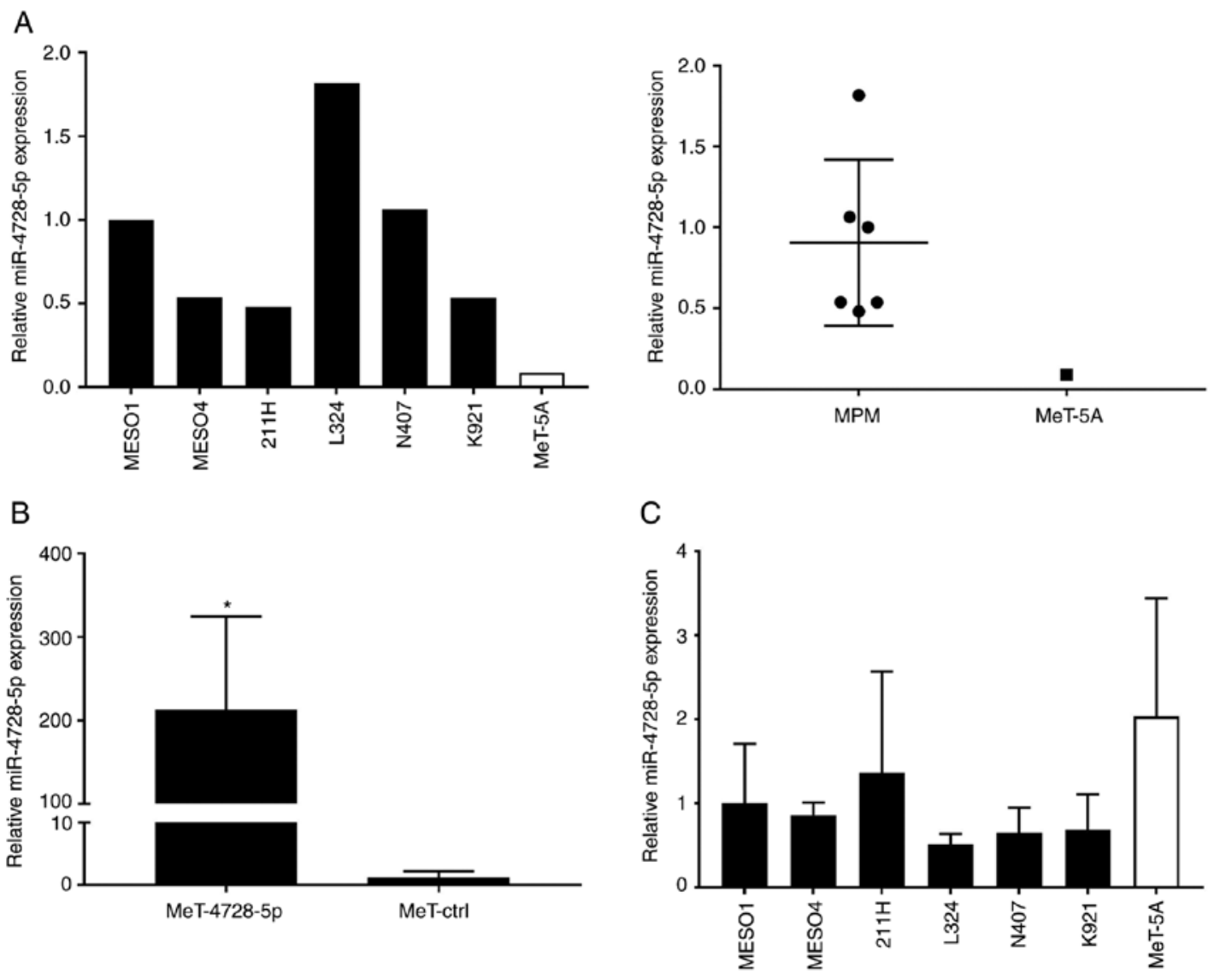

Figure 5. Expression analysis of hsa-miR-4728-5p. (A) Expression levels of hsa-miR-4728-5p in EV50s isolated from six MPM cell lines and MeT-5A cells analyzed by qPCR. The left and right panels show the expression level of each cell line and the comparison between MPM and MeT-5A cells, respectively. Expression was corrected by U6 counts, and expression level of ACC-MESO1 cells was set to 1. (B) MeT-5A cells overexpressing hsa-miR-4728-5p (MeT-4728-5p) and control miRNA (MeT-ctrl) were established. Each intracellular expression level was measured by qPCR and corrected by U6 counts. The expression level of MeT-ctrl was set to 1 . The experiment was performed twice independently; bars, SD. * $\mathrm{P}<0.05$. (C) Intracellular expression levels of hsa-miR-4728-5p in each cell line were analyzed by qPCR and corrected by U6 counts. The expression level of ACC-MESO1 cells was set to 1 . MPM, malignant pleural mesothelioma; EV50s, EVs captured by a 50 -nm filter.

that EVs in the medium could be taken up by other cells. To quantify extracellular secretion and endocytosis of EVs, nanoluciferase (Nluc) fusion proteins, CD63-Nluc, CD9-Nluc, and CD81-Nluc were expressed in cells and analyzed. The amount of extracellular secretion of each Nluc fusion protein was found to be increased in a time-dependent manner (Fig. 3A). Since the luciferase activity of CD81-Nluc was the highest among the three, the capture rate of the syringe filter was calculated using cells that expressed CD81-Nluc. As shown in Fig. 3B, 64\% of EVpas220 were captured at $20 \mathrm{~nm}$, of which $80 \%$ of the EVs were captured at $50 \mathrm{~nm}$. Next, we investigated whether MeT-5A and IHPF cells were able to take up EVs with diameters between 50 and $220 \mathrm{~nm}$. As shown in Fig. 3C, EV uptake increased in a time-dependent manner, but the amount varied between cells.

Microarray analysis with EV50-miRNA. As shown in Fig. 3B, EVcap50/220 captured $80 \%$ of EVs with diameters between 20 and $220 \mathrm{~nm}$, which included exosomes and microvesicles, but not apoptotic bodies. Considering the collection efficiency and filter handling (pressure power, flow volume), miRNAs in EVs were purified from EV50/220 (EV50-miRNAs). Expression profiles of the EV50-miRNAs were obtained by a 3D-Gene microarray system, and were compared between 6 types of malignant pleural mesothelioma (MPM) and MeT-5A cells. This chip contained a set of 2,565 probes derived from human cells, and signals detected for each miRNA were normalized by a global normalization method that adjusted the median of the detected signal intensity to 25 . From the results of the microarray, 390 and 502 EV50-miRNAs were detected in MeT-5A and all six MPM cell lines, respectively. As shown in Fig. 4A, 11 EV50-miRNAs were detected exclusively in MeT-5A cells, but not in MPM cells. On the other hand, 123 EV50-miRNAs were detected in MPM cells but not in MeT-5A cells. These EV50-miRNAs may be markers of MPM. Among the 123 EV50-miRNAs, the 20 most common variants are shown in Table I in descending order of expression. The ratio was calculated using the mean values of MPM cells. Expression of these EV50-miRNAs are also shown on a heat map (Fig. 4B). Since the expression value of hsa-miR-4728-5p was the highest among all EV50-miRNAs, we focused on this miRNA and searched for its target genes.

Search for targets of hsa-miR-4728-5p in MeT-5A cells. First, we confirmed the expression of hsa-miR-4728-5p in EVcap50/220 of MPM and MeT-5A cells by qPCR. As shown in Fig. 5A, expression levels of hsa-miR-4728-5p in MPM cells 

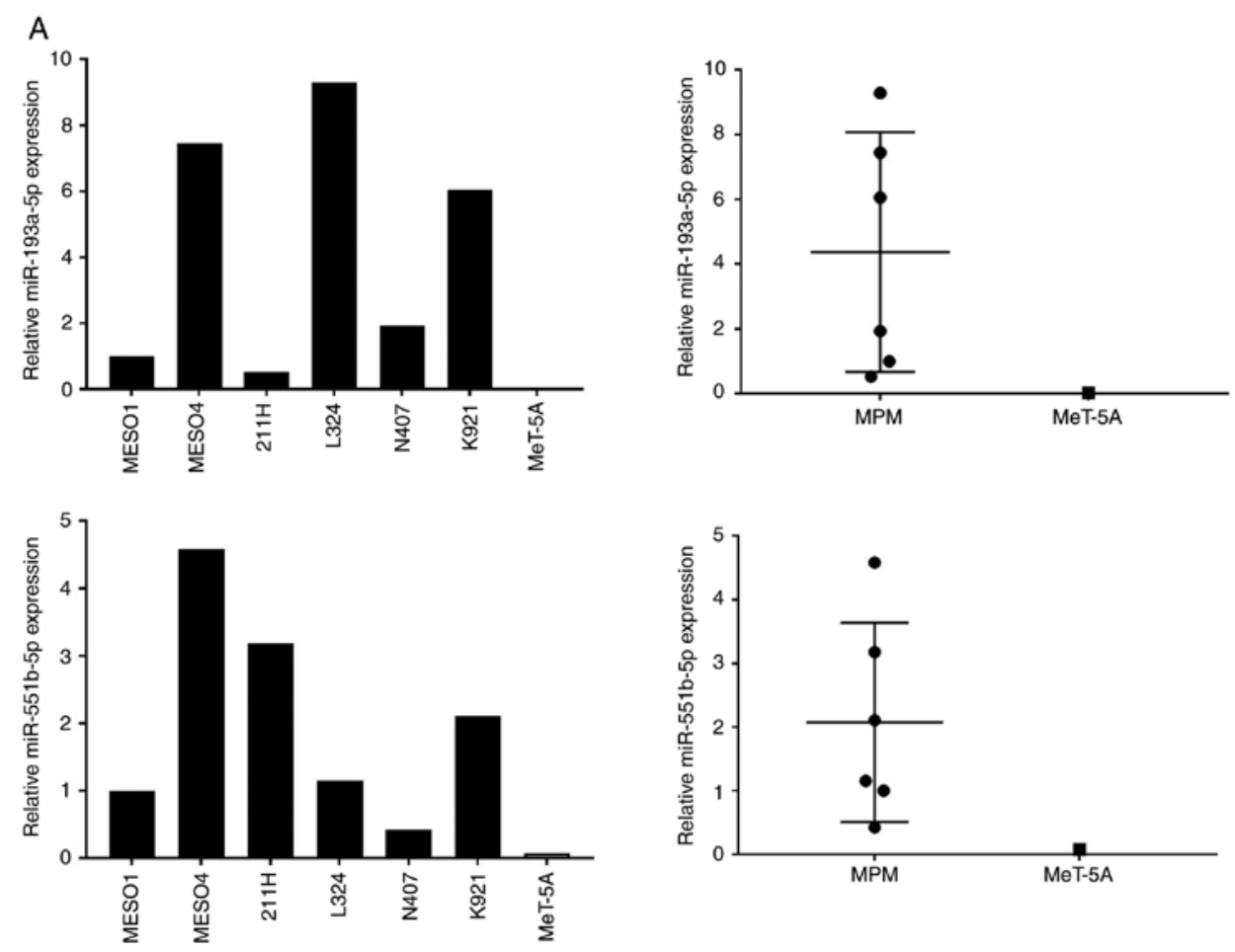

B
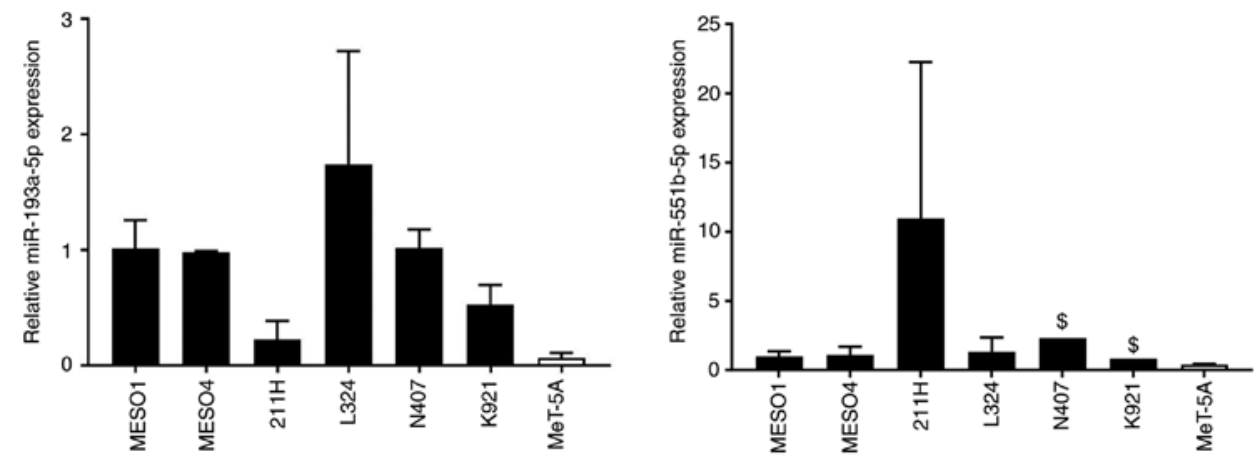

Figure 6. Expression analysis of hsa-miR-193a-5p and hsa-miR-551b-5p. (A) Expression levels of miR-193a-5p and miR-551b-5p in EV50s isolated from six MPM cell lines and MeT-5A cells were analyzed by qPCR. The left and right panels show the expression level of each cell line and the comparison between MPM and MeT-5A cells, respectively. Expression was corrected by U6 counts, and expression level of ACC-MESO1 cells was set to 1. (B) Intracellular expression levels of hsa-miR-193a-5p and hsa-miR-551b-5p were analyzed by qPCR. The experiment was performed two times independently. Expression was corrected by U6 counts, and expression level of ACC-MESO1 cells was set to 1. \$ indicate that standard deviation could not be calculated because at least one value was below the detection limit. MPM, malignant pleural mesothelioma; EV50s, EVs captured by a 50-nm filter.

were 5-17 times higher as compared with those of MeT-5A cells. To investigate the target genes of hsa-miR-4728-5p in normal cells, we established MeT-5A cells that overexpressed this miRNA (MeT-4728 cells) as well as control miRNA-free cells (MeT-ctrl). Since it was confirmed that hsa-miR-4728-5p was overexpressed in the MeT-4728-5p cells (Fig. 5B), microarray analysis was performed using mRNA obtained from MeT-4728-5p and MeT-ctrl cells. Based on the result, only two mRNAs, which came from EIF4H and TMEM87A, satisfied the following two conditions: i) Expression level of genes after global normalization was higher than 100 in MeT-ctrl cells; ii) expression levels of genes in MeT-4728 cells decreased to less than 0.5 when compared to MeT-ctrl cells. Since there are few mRNAs whose expression is suppressed by hsa-miR-4728-5p, the intracellular expression of hsa-miR-4728-5p in each cell was analyzed by qPCR. Contrary to our expectations, its expression was found to be highest in MeT-5A cells (Fig. 5C). These data suggest that MeT-5A cells express hsa-miR-4728-5p, but do not secrete it extracellularly, and function only in the cell.

Search for targets of hsa-miR-193a-5p and hsa-miR-551b-5p in MeT-5A cells. As described above, hsa-miR-4728-5p, which showed the highest expression in EV50-miRNAs of MPM cells, was highly expressed in MeT-5A cells. Therefore, we selected EV50-miRNAs that were not found inside MeT-5A cells from 123 EV50-miRNAs via microarray analysis. As a result, 6 miRNAs were listed as candidates (Table II). Based on these results, we selected hsa-miR-193a-5p and hsa-miR-551b-5p, which exhibited the highest expression. It was confirmed that these miRNAs were contained in EV50-miRNAs of many MPM cells, and were not often present in the MeT-5A cells (Fig. 6A). We also examined the expression of hsa-miR-193a-5p and hsa-miR-551b-5p in 
A
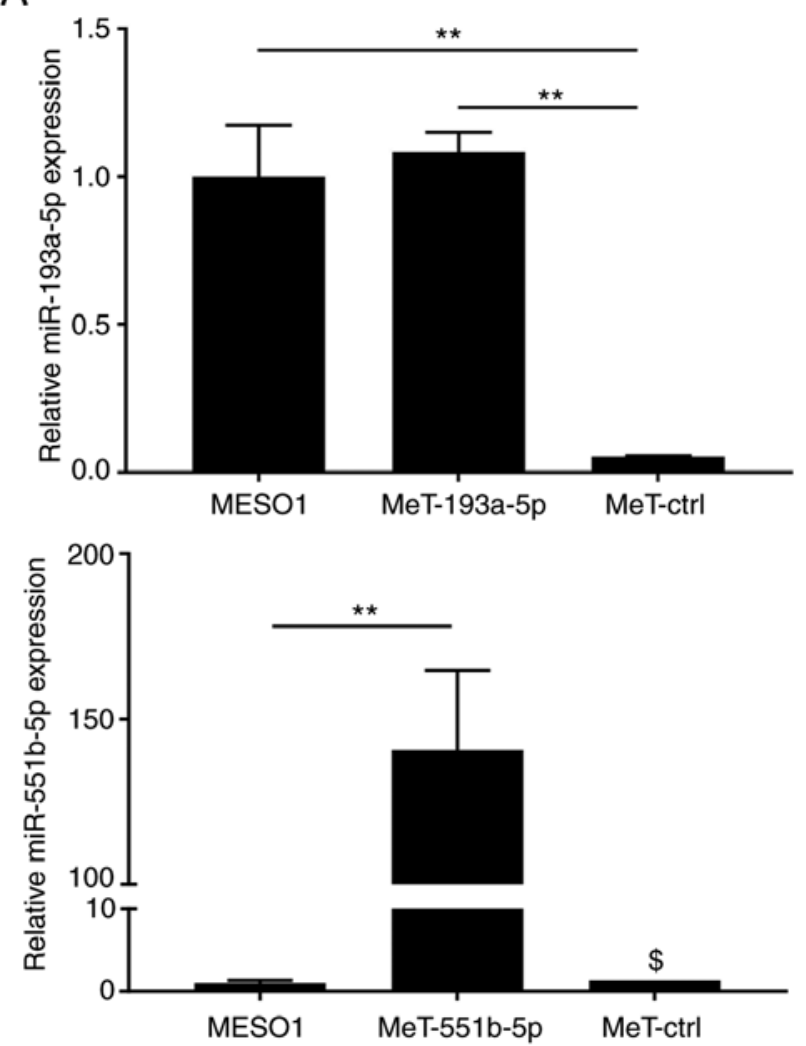

B

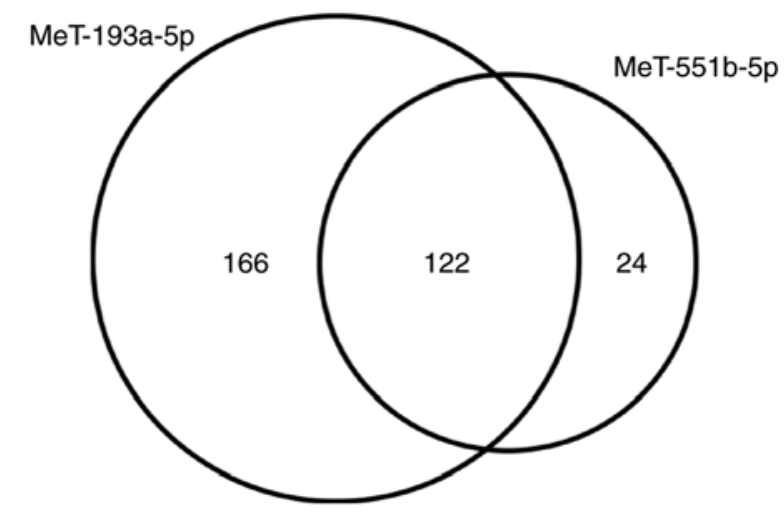

Figure 7. Target gene analysis of hsa-miR-193a-5p and hsa-miR-551b-5p. (A) MeT-5A cells overexpressing hsa-miR-193a-5p (MeT-193a-5p) or hsa-miR-551b-5p (MeT-551b-5p) were established. Each intracellular expression level was measured by qPCR and corrected by U6 counts. The expression level of ACC-MESO1 cells were set to 1 . The experiment was performed twice independently; bars, SD. ${ }^{* *} \mathrm{P}<0.01$. ${ }^{\text {IIndicate that standard }}$ deviation could not be calculated because at least one value was below the detection limit. (B) Number of mRNAs commonly or specifically repressed in MeT-193a-5p and MeT-551b-5p cells, as shown in a Venn diagram.

cells, but found no correlation in miRNA expression between MPM and MeT-5A cells (Fig. 6A and B). Next, we established MeT-5A cells that overexpressed hsa-miR-193a-5p (MeT-193a cells) or hsa-miR-551b-5p (MeT-551b cells) to investigate their influence on gene expression (Fig. 7A). There were 288 mRNAs in the MeT-193a-5p cells and 146 mRNAs in the MeT-551b-5p cells that satisfied the following two conditions: i) Expression levels of genes after global normalization were higher than 100 in MeT-ctrl cells; ii) expression levels of genes in MeT-193a-5p cells or MeT-551b-5p cells decreased to less than 0.2 when compared to MeT-ctrl cells. Interestingly, the expression of 122 mRNAs was found to be suppressed (Fig. 7B). The function of these genes was analyzed with the GeneCoDis3 software (http://genecodis.cnb.csic.es/), and it was found that these genes are involved in several pathways (Table III). Among these functions, we focused on genes involved in the binding between cells as well as between the cell and the extracellular matrix, as MPM cells may reduce the adhesion ability of normal mesothelial cells. Invading the space between these cells is important for malignant transformation. A summary of each gene is shown in Table IV.

\section{Discussion}

Malignant pleural mesothelioma (MPM) is a disease associated with a poor patient prognosis, and the development of diagnostic markers for early detection is urgently required. Since initial studies have shown that extracellular vesicles (EVs) can mediate intercellular transfer of RNA and proteins (12-15), many studies have focused on the contents of EVs, and have tried to deduce their involvement in intercellular communications $(21,22)$. The purpose of this study was to compare the profiles of EV-derived miRNAs between MPM cell lines and a non-malignant cell line, and to propose microRNAs (miRNAs) that would be useful in diagnosing MPM.

Recently, EVs have attracted great attention in both therapeutic and diagnostic applications (23-26). To isolate EVs such as exosomes, there are various methods, including ultracentrifugation, commercial kits that use antibodies against exosome membrane surface proteins, and size-exclusion chromatography (27-29). These methods have advantages and disadvantages in terms of steps and time. We should keep in mind the possibility that EVs could influence analyses because it is difficult to exclude the captured substances and eluates using antibodies against exosome membrane surface proteins. When using these to isolate EVs from liquids, a choice must be made based on the advantages and disadvantages of the method. In the present study, we employed filter membranes to isolate EVs based on their diameters. This method is simple and logical because EVs such as exosomes, microvesicles, and apoptotic bodies are classified by diameter. A $50-\mathrm{nm}$ membrane filter could capture $80 \%$ of EVs that passes through a 220-nm membrane filter. We believe that this capture rate is sufficient for analyzing RNAs contained in exosomes and microvesicles. The EVs captured by a $50-\mathrm{nm}$ filter membrane were analyzed using dynamic light scattering (DLS), transmission electron microscopy (TEM), western blot analysis, and BioAnalyzer 2100. Based on our analysis on diameter, form, specific proteins, and inclusion of miRNAs, the isolated EVcap50/220 and EVrev50/220 were considered structurally to be EVs. Furthermore, endocytosis analysis of EVs with diameters between 50 and $220 \mathrm{~nm}$ found them to be functionally EVs. Therefore, we consider this filter method to be useful for EV analysis because it can not only capture EVs in liquid, but also easily remove EVs from liquid. For example, we removed EVs derived from fetal bovine serum (FBS) using this method. This means that EVs can be easily removed from the cell maintenance medium used in the laboratory.

MPM originates in pleura mesothelial cells, and many MPM patients have pleural effusion. We speculated that EVs 
Table III. Pathway analysis of hsa-miR-193a-5p and hsa-miR-551b-5p target genes (GeneCodis analysis, KEGG pathways).

\begin{tabular}{|c|c|c|c|c|}
\hline No. of genes & NGR & Hyp & Нyр* & Annotations \\
\hline 8 & 197 & $3.20 \mathrm{E}-07$ & $2.82 \mathrm{E}-05$ & Focal adhesion \\
\hline 4 & 71 & $9.25 \mathrm{E}-05$ & $2.71 \mathrm{E}-03$ & Adherens junction \\
\hline 5 & 149 & $1.41 \mathrm{E}-04$ & $3.10 \mathrm{E}-03$ & Wnt signaling pathway \\
\hline 4 & 67 & 7.37E-05 & $3.24 \mathrm{E}-03$ & p53 signaling pathway \\
\hline 4 & 102 & $3.74 \mathrm{E}-04$ & $6.58 \mathrm{E}-03$ & Amoebiasis \\
\hline 4 & 113 & $5.51 \mathrm{E}-04$ & $8.08 \mathrm{E}-03$ & Leukocyte transendothelial migration \\
\hline 4 & 130 & $9.31 \mathrm{E}-04$ & $1.02 \mathrm{E}-02$ & Tight junction \\
\hline 4 & 128 & 8.79E-04 & $1.10 \mathrm{E}-02$ & Axon guidance \\
\hline 3 & 68 & $1.50 \mathrm{E}-03$ & $1.47 \mathrm{E}-02$ & Long-term potentiation \\
\hline 3 & 82 & $2.57 \mathrm{E}-03$ & $2.26 \mathrm{E}-02$ & TGF- $\beta$ signaling pathway \\
\hline 4 & 186 & $3.44 \mathrm{E}-03$ & $2.75 \mathrm{E}-02$ & Chemokine signaling pathway \\
\hline 3 & 98 & 4.25E-03 & $2.88 \mathrm{E}-02$ & GnRH signaling pathway \\
\hline 3 & 98 & $4.25 \mathrm{E}-03$ & $2.88 \mathrm{E}-02$ & Melanogenesis \\
\hline 2 & 33 & $5.34 \mathrm{E}-03$ & $3.36 \mathrm{E}-02$ & African trypanosomiasis \\
\hline 3 & 113 & $6.31 \mathrm{E}-03$ & $3.70 \mathrm{E}-02$ & Vascular smooth muscle contraction \\
\hline 2 & 42 & $8.54 \mathrm{E}-03$ & 4.70E-02 & Aldosterone-regulated sodium reabsorption \\
\hline
\end{tabular}

hsa, human (Homo sapiens); KEGG, Kyoto Encyclopedia of Genes and Genomes; NGR, number of annotated genes in the reference list; Hyp, hypergeometric P-value; Hyp*, corrected hypergeometric P-value.

Table IV. Suppression of genes associated with cell-cell and cell-matrix adhesion by hsa-miR-913a-5p and hsa-miR-551b-5p.

\begin{tabular}{|c|c|c|c|c|c|}
\hline Gene & $\begin{array}{c}\text { Genes } \\
\text { suppressed by } \\
\text { miR-193a-5p }\end{array}$ & $\begin{array}{c}\text { Genes } \\
\text { suppressed by } \\
\text { miR-551b-5p }\end{array}$ & $\begin{array}{l}\text { Genes associated } \\
\text { with focal adhesion }\end{array}$ & $\begin{array}{c}\text { Genes associated } \\
\text { with tight } \\
\text { junction }\end{array}$ & $\begin{array}{c}\text { Genes associated } \\
\text { with adherens } \\
\text { junction }\end{array}$ \\
\hline$A C T N 1$ & 0 & O & O & O & 0 \\
\hline$P R K C B$ & O & 0 & O & O & \\
\hline$C C N D 1$ & 0 & & ○ & & \\
\hline THBS1 & O & o & O & & \\
\hline CAPN2 & O & O & 0 & & \\
\hline$C A V 1$ & O & O & 0 & & \\
\hline$L A M B 3$ & o & o & O & & \\
\hline$J U N$ & o & o & ० & & \\
\hline$R O C K 1$ & 0 & 0 & O & & \\
\hline$F L N B$ & & O & O & & \\
\hline PARD3 & O & o & & o & o \\
\hline MLLT4 & O & O & & o & O \\
\hline$P P P 2 R 1 B$ & O & & & O & \\
\hline CREBBP & O & 0 & & & O \\
\hline
\end{tabular}

secreted from MPM cells exist in both the blood and pleural effusion. Therefore, we used a culture medium that resembles pleural effusion. We performed miRNA array analyses using EV50-miRNAs of six MPM cell lines and normal pleura mesothelial cells (MeT-5A) to select specific EV50-miRNAs for each cell. Since it is more convenient to test for EV50-miRNAs that proliferate in MPM cells for diagnosis, we focused on EV50-miRNAs that could be detected only in MPM cells. 
There were 123 EV50-miRNAs that were present exclusively in MPM-derived EVs when they underwent microarray analysis. It was speculated that EVs secreted by MPM cells are taken up by normal mesothelial cells or fibroblasts, and MPM-derived miRNAs regulate the gene expression of these cells. For this reason, we selected the most highly expressed miRNA, hsa-miR-4728-5p, to be overexpressed in MeT-5A cells. Contrary to expectations, most mRNA expression levels did not vary, and overexpression of hsa-miR-4728-5p reduced only two genes to less than 0.5 compared to the control cells. We hypothesized that hsa-miR-4728-5p is heavily expressed inside MeT-5A cells but has little effect on the suppression of genes.

Even if a cell synthesizes a particular miRNA and does not encapsulate it in EVs under normal conditions, it is possible that the miRNA may be encapsulated in EVs under other environmental conditions (inflammation, etc.) and secreted. Therefore, we thought that it was necessary to select miRNAs that were not synthesized in normal cells. Excluding the miRNAs that are synthesized in MeT-5A cells from the original 123 EV50-miRNAs derived from MPM cells, six miRNAs remained.

Cancer cells secrete EVs containing exosomes, incorporate them into normal cells, and use internal mRNAs and miRNAs to alter the gene expression of normal cells. This suggested that cancer cells changed the microenvironment to be more favorable for them. It has been reported that when exosomes containing MMP1 (matrix metalloproteinase 1) mRNA are secreted from ovarian cancer cells and taken up by normal peritoneal mesothelial cells, MMP1 mRNA induces apoptosis in these healthy cells and contributes to ovarian cancer metastasis (30). In order to investigate the effect of EV50-miRNA uptake on gene expression of cells, MeT-5A cells overexpressing hsa-miR-193a-5p or hsa-miR-551b-5p were established. These two EV50-miRNAs were selected as they were highly expressed in MPM cells but not in normal pleural mesothelial cells (MeT-5A cells). Since these two EV50-miRNAs reduced thousands of genes to less than 0.5 when compared to control cells, genes were selected by narrowing down the selection margin to less than 0.2. A few hundreds of genes were selected, and despite the fact that they came from reads of two different miRNAs, they suppressed the expression of many common genes. Suppression of mRNA expression by miRNAs is permanent rather than transient, so both primary (direct) and secondary (indirect) suppression are included in our study. Genes whose expression was commonly suppressed by hsa-miR-193a-5p and hsa-miR-551b-5p were analyzed using pathways from the Kyoto Encyclopedia of Genes and Genomes (KEGG). Many genes were found to be involved in cell-cell and cell-matrix adhesion. For cancer cells to invade normal tissues, it is necessary to destroy cell-cell and cell-matrix adhesion, and there have been many studies that suggest that secreted or membrane-bound types of MMPs are capable of digesting matrix proteins (31). Therefore, decreasing the expression of proteins associated with tight junctions, adherens junctions, and focal adhesion may contribute to successful invasion of MPM cells. Zhou et al (32) reported that PARD3 contributes to the epithelial-mesenchymal transition (EMT) and invasion of non-small-cell lung cancer (NSCLC). PARD3 is associated with tight junctions and adherens junctions, and our results showed that PARD3 mRNA could be repressed by both hsa-miR-193a-5p and hsa-miR-551b-5p.

Tight junctions are one of the intercellular adhesion structures that control traffic of substances between normal cells (33). Tight junctions also play an important role in cancer cells. Downregulation or loss of tight junctions can both contribute to cancer progression by altering cell migration, proliferation, and differentiation (34-37). In current studies, reduction of tight junction-associated $\mathrm{ZO}-1$ in breast tumors was associated with metastasis in breast cancers (38). In addition, Zhou et al (9) showed that cancer-secreted miR-105 was capable of suppressing ZO-1 and promoting metastasis in breast cancers. In our study, hsa-miR-193a-5p and hsa-miR-551b-5p suppressed genes associated with tight junctions, which may contribute to metastasis in MPM cells.

Many studies have suggested that EVs may be attractive targets for both therapeutic and diagnostic applications (23-26). Previously, proteomic analysis of EVs isolated from human malignant pleural effusions has been reported (39), but there are no reports on miRNA-based analyses of EVs. Our results indicated that six EV50-miRNAs in blood and pleural effusions may serve as novel diagnostic markers for MPM. In particular, since the expression of hsa-miR-193a-5p and hsa-miR-551b-5p are high in MPM-derived EVs, these are very promising diagnostic markers. To date, there have been no reports of using EV-derived hsa-miR-193a-5p and hsa-miR-551b-5p for MPM diagnoses. Plasma-derived and serum-derived hsa-miR-193a-5p have been reported as promising indicators for diagnosing amyotrophic lateral sclerosis (ALS) (40), ovarian cancer (41), chronic myeloid leukemia (42), human T-cell leukemia virus type 1 (HTLV-1) infection (43), colorectal cancer metastasis (44), and aortic aneurysms (45). On the other hand, plasma-derived or serum-derived hsa-miR-551b-5p has been reported as a promising indicator of acute pancreatitis $(46,47)$ and gastric cancer (48). As far as we are aware, there are no reports of using EV-derived miRNAs to diagnose MPM, and our report will be the first to do so.

In this experiment, miRNAs were selected from the microarray results and verified by qPCR, but there was no correlation in the expression ratio between cells in each analysis. It has been reported that miRNA expression does not necessarily correlate between qPCR and microarrays (49). The major difference is that qPCR amplifies miRNA samples, but microarray analysis does not. What is important is that the results between tumor and normal cells were trend-matched and not reversed between qPCR and the microarray in this study.

In summary, we showed that many EVs could be easily isolated and removed from liquids using 50 and $220-\mathrm{nm}$ membrane filters. Using this method, we identified six miRNAs that could be specifically secreted from MPM cells and used to diagnose MPM. Among them, hsa-miR-193a-5p and hsa-miR-551b-5p strongly inhibited expression of genes related to cell-cell interactions and cell-matrix interactions in normal cells. These results suggest that hsa-miR-193a-5p and hsa-miR-551b-5b may contribute to invasion of MPM cells, and may help elucidate the mechanism of malignant acquisition of MPM. Since this study is an in vitro analysis, 
verification analysis using clinical specimens is necessary in the future.

\section{Acknowledgements}

We would like to thank Yukiko Yoshiura (Center for Stress-related Disease Control and Prevention, University of Occupational and Environmental Health, Japan.) for her technical assistance.

\section{Funding}

This research received no external funding.

\section{Availability of data and materials}

The datasets used and analyzed during the current study are available from the corresponding author on reasonable request. Supplementary materials have been provided by the authors and are published online. They are linked to our website and can be accessed by the reader.

\section{Authors' contributions}

Conceptualization of the study was accomplished by HI. Formal analysis was carried out by TJ. Methodology was designed by HI. Project administration of the experiments was carried out by YM and KY. Supervision of the research was the responsibility of YM and KY. Writing of the original draft was carried out by TJ and writing and review and edited was conducted by HI. All authors read and approved the manuscript and agree to be accountable for all aspects of the research in ensuring that the accuracy or integrity of any part of the work are appropriately investigated and resolved.

\section{Ethics approval and consent to participate}

Not applicable.

\section{Patient consent for publication}

Not applicable.

\section{Competing interests}

The authors declare that they have no competing interests.

\section{References}

1. Robinson BM and Lake RA: Advances in malignant mesothelioma. N Engl J Med 353: 1591-1603, 2005.

2. Robinson BM: Malignant pleural mesothelioma: An epidemiological perspective. Ann Cardiothorac Surg 1: 491-496, 2012.

3. Jamrozik E, de Klerk N and Musk AW: Asbestos-related disease. Intern Med J 41: 372-380, 2011.

4. Creaney J and Robinson BW: Serum and pleural fluid biomarkers for mesothelioma. Curr Opin Pulm Med 15: 366-370, 2009.

5. Pass HI, Lott D, Lonardo F, Harbut M, Liu Z, Tang N, Carbone M, Webb C and Wali A: Asbestos exposure, pleural mesothelioma, and serum osteopontin levels. N Engl J Med 353: 1564-1573, 2005.

6. Ren R, Yin P, Zhang Y, Zhou J,Zhou Y, Xu R, Lin H and Huang C: Diagnostic value of fubulin-3 for malignant leural mesothelioma: A systematic review and meta-analysis. Oncotarget 7: 84851-84859, 2016
7. Zhang L, Zhang S, Yao J, Lowery FJ, Zhang Q, Huang WC, Li P, Li M, Wang X, Zhang C, et al: Microenvironment-induced PTEN loss by exosomal microRNA primes brain metastasis outgrowth. Nature 527: 100-104, 2015.

8. Harazono Y, Muramatsu T, Endo H, Uzawa N, Kawano T, Harada K, Inazawa J and Kozaki K: miR-655 Is an EMT-suppressive microRNA targeting ZEB1 and TGFBR2. PLoS One 8: e62757, 2013.

9. Zhou W, Fong MY, Min Y, Somlo G, Liu L, Palomares MR, Yu Y, Chow A, O'Connor ST, Chin AR, et al: Cancer-secreted miR-105 destroys vascular endothelial barriers to promote metastasis. Cancer Cell 25: 501-515, 2014.

10. Gee GV, Koestlr DC, Christensen BC, Sugarbaker DJ, Ugolini D, Ivaldi GP, Resnick MB, Houseman EA, Kelsey KT and Marsit CJ: Downregulated microRNAs in the differential diagnosis of malignant pleural mesothelioma. Int J Cancer 127: 2859-2869, 2010.

11. Juri A, Zanoaga O, Braicu C, Tomuleasa C, Irimie A and Berindan-Neagoe I: A comprehensive picture of extracellular vesicles and their contents. Molecular transfer to cancer cells. Cancers (Basel) 12: 298, 2020.

12. Valadi H, Ekström K, Bossios A, Sjöstrand M, Lee JJ and Lötvall JO: Exosome-mediated transfer of mRNAs and microRNAs is a novel mechanism of genetic exchange between cells. Nat Cell Biol 9: 654-659, 2007.

13. Pegtel DM, Cosmopoulos K, Thorley-Lawson DA, van Eijndhoven MA, Hopmans ES, Lindenberg JL, de Gruijl TD, Würdinger T and Middeldorp JM: Functional delivery of viral miRNAs via exosomes. Proc Natl Acad Sci USA 107: 6328-6333, 2010.

14. Zhang Y, Liu D, Chen X, Li J, Li L, Bian Z, Sun F, Lu J, Yin Y, Cai X, et al: Secreted monocytic miR-150 enhances targeted endothelial cell migration. Mol Cell 39: 133-144, 2010.

15. Kosaka N, Iguchi H, Yoshioka Y, Takeshita Y, Matsuki Y and Ochiya T: Secretory mechanisms and intercellular transfer of microRNAs in living cells. J Biol Chem 285: 17442-17452, 2010.

16. Yasuda M, Hanagiri T, Shigematsu Y, Onitsuka T, Kuroda K, Baba T, Mizukami M, Ichiki Y, Uramoto H, Takenoyama M and Yasumoto K: Identification of a tumor associated antigen in lung cancer patients with asbestos exposure. Anticancer Res 30: 2631-2639, 2010

17. Koi C, Izumi H, Kurita T, Nguyen TT, Murakami M, Yoshiura Y, Hachisuga T and Morimoto Y: Lovastatin induced Kruppel like factor 2 (KLF2), Kruppel like factor 6 (KLF6) and Ras homolog family member B (RHOB) genes and preferentially led to viability reduction of Cisplatin-resistant cells. Oncotarget 8: 106429-106442, 2017.

18. Morimoto $\mathrm{Y}$, Izumi $\mathrm{H}$, Yoshiura $\mathrm{Y}$, Tomonaga $\mathrm{T}$, Oyabu $\mathrm{T}$, Myojo T, Kawai K, Yatera K, Shimada M, Kubo M, et al: Pulmonary toxicity of well-dispersed cerium oxide nanoparticles following intratracheal instillation and inhalation. J Nanopart Res 17: 442, 2015.

19. Samadder P, Byun HS, Bittman R and Arthur G: A fluorescent alkyllysophospholipid analog exhibits selective cytotoxicity against the hormone-insensitive prostate cancer cell line PC3. Anticancer Agents Med Chem 14: 528-538, 2014.

20. Jabbari N, Akbariazar E, Feqhhi M, Rahbarghazi R and Rezaie J: Breast cancer-derived exosomes: Tumor progression and therapeutic agents. J Cell Physiol 235: 6345-6356, 2020.

21. Guo Y, Ji X, Liu J, Fan D, Zhou Q, Chen C, Wang W, Wang G, Wang H, Yuan W, et al: Effects of exosomes on pre-metastatic formation in tumors. Mol Cancer 18: 39, 2019.

22. Kling CM: Non-coding RNAs in breast cancer: Intracellular and intercellular communication. Noncording RNA 4: 40, 2018.

23. Jayaseelan VP: Emerging role of exosomes as promising diagnostic tool for cancer. Cancer Gene Ther 27: 395-398, 2020.

24. Wu Z, Yang Z, Dai Y, Zhu Q and Chen LA: Update on liquid biopsy in clinical management of non-small cell lung cancer. Onco Targets Ther 12: 5097-5109, 2019.

25. Fortunato O, Gasparini P, Boeri M and Sozzi G: Exo-miRNAs as a new tool for liquid biopsy in lung cancer. Cancers (Basel) 11: 888,2019

26. Jiang L, Gu Y, Du Y and Liu J: Exosomes: Diagnostic biomarkers and therapeutic delivery vehicles for cancer. Mol Pharm 16: 3333-3349, 2019.

27. Purushothaman A: Exosomes from cell culture-conditioned medium: Isolation by ultracentrifugation and characterization. Methods Mol Biol 1952: 233-244, 2019. 
28. Skottvoll FS, Berg HE, Bjørseth K, Lund K, Roos N, Bekhradnia S, Thiede B, Sandberg C, Vik-Mo EO, Roberg-Larsen H, et al: Ultracentrifugation versus kit exosome isolation: nanoLC-MS and other tools reveal similar performance biomarkers, but also contaminations. Future Sci OA 5: FSO359, 2018

29. Baranyai T, Herczerg K, Onódi Z, Voszka I, Módos K, Marton N, Nagy G, Mäger I, Wood MJ, EI Andaloussi S, et al: Isolation of exosomes from blood plasma: Qualitative and quantitative comparison of ultracentrifugation and size exclusion chromatography methods. PLoS One 10: e0145686, 2015.

30. Yokoi A, Yoshioka Y, Yamamoto Y, Ishikawa M, Ikeda SI, Kato T, Kiyono T, Takeshita F, Kajiyama H, Kikkawa F and Ochiya T: Malignant extracellular vesicles carrying MMP1 mRNA facilitate peritoneal dissemination in ovarian cancer. Nat Commun 8: 14470, 2017.

31. Huang H: Matrix metalloproteinase-9 (MMP-9) as a cancer biomarker and MMP-9 biosensors: Recent advances. Sensors (Basel) 18: 3249, 2018.

32. Zhou Q, Dai J, Chen T, Dada LA, Zhang X, Zhang W, DeCamp MM, Winn RA, Sznajder JI and Zhou G: Downregulation of PKC /Pard3/Pard6b is responsible for lung adenocarcinoma cell EMT and invasion. Cell Signal 38: 49-59, 2017.

33. Tsukita S, Furuse M and Itoh M: Multifunctional strands in tight junctions. Nat Rev Mol Cell Biol 2: 285-293, 2001.

34. Brennan K, Offiah G, McSherry EA and Hopkins AM: Tight junctions: A barrier to the initiation and progression of breast cancer? J Biomed Biotechnol 2010: 460607, 2010.

35. Georgiadis A, Tshernutter M, Bainbridge JW, Balaggan KS, Mowat F, West EL, Munro PM, Thrasher AJ, Matter K, Balda MS and Ali RR: The tight junction associated signalling proteins ZO-1 and ZONAB regulate retinal pigment epithelium homeostasis in mice. PLoS One 5: e15730, 2010.

36. Itoh $\mathrm{M}$ and Bissell $\mathrm{MJ}$ : The organization of tight junctions in epithelia: Implications for mammary gland biology and breast tumorigenesis. J Mammary Gland Biol Neoplasia 8: 449-462, 2003.

37. Martin TA and Jiang WG: Loss of tight junction barrier function and its role in cancer metastasis. Biochim Biophys Acta 1788: 872-891, 2009

38. Polette M, Gilles C, Nawrocki-Raby B, Lohi J, Hunziker W, Foidart JM and Birembaut P: Membrane-type 1 matrix metalloproteinase expression is regulated by zonula occludens-1 in human breast cancer cells. Cancer Res 65: 7691-7698, 2005.

39. Bard MP, Hegmans JP, Hemmes A, Luider TM, Willemsen R, Severijnen LA, van Meerbeeck JP, Burgers SA, Hoogsteden HC and Lambrecht BN: Proteomic analysis of exosomes isolated from human malignant pleural effusions. Am J Respir Cell Mol Biol 31: 114-121, 2004.
40. Saucier D, Wajnberg G, Roy J, Beauregard AP, Chacko S, Crapoulet N, Fournier S, Ghosh A, Lewis SM, Marrero A, et al: Identification of a circulating miRNA signature in extracellular vesicles collected from amyotrophic lateral sclerosis patients. Brain Res 1708: 100-108, 2019.

41. Ren X, Zhang H, Cong H, Wang X, Ni H, Shen X and Ju S Diagnostic model of serum miR-193a-5p, HE4 and CA125 improves the diagnostic efficacy of epithelium ovarian cancer. Pathol Oncol Res 24: 739-744, 2018.

42. Prinsloo A, Pool R and Van Niekerk C: Preliminary data on microRNA expression profiles in a group of South African patients diagnosed with chronic myeloid leukaemia. Mol Clin Oncol 7: 386-390, 2017

43. Fayyad-Kazan M, EIDirani R, Hamade E, EI Majzoub R, Akl H, Bitar N, Fayyad-Kazan H and Badran B: Circulating miR-29c, miR-30c, miR-193a-5p and miR-885-5p: Novel potential biomarkers for HTLV-1 infection diagnosis. Infect Genet Evol 74: 103938, 2019.

44. Qu A, Yang Y, Zhang X, Wang W, Liu Y, Zheng G, Du L and Wang C: Development of a preoperative prediction nomogram for lymph node metastasis in colorectal cancer based on a novel serum miRNA signature and CT scans. EBioMedicine 37: $125-133,2018$

45. Moushi A, Michalidou K, Soteriou M, Cariolou M and Bashiardes E: MicroRNAs as possible biomarkers for screening of aortic aneurysms: A systematic review and validation study. Biomarkers 23: 253-264, 2018.

46. Zhang Y, Yan L and Han W: Elevated level of miR-551b-5p is associated with inflammation and disease progression in patients with severe acute pancreatitis. Ther Apher Dial 22: 649-655, 2018.

47. Liu P, Xia L, Zhang WL, Ke HJ, Su T, Deng LB, Chen YX and Lv NH: Identification of serum microRNAs as diagnostic and prognostic biomarkers for acute pancreatitis. Pancreatology 14: 159-166, 2014

48. Jiang X, Jiang M, Xu M, Xu J and Li Y: Identification of diagnostic utility and molecular mechanisms of circulating miR-551b-5p in gastric cancer. Pathol Res Pract 215: 900-904, 2019.

49. Chen Y, Gelfond JA, McManus LM and Shireman PK: Reproducibility of quantitative RT-PCR array in miRNA expression profiling and comparison with microarray analysis. BMC Genomics 10: 407, 2009.

This work is licensed under a Creative Commons Attribution-NonCommercial-NoDerivatives 4.0 International (CC BY-NC-ND 4.0) License. 